\section{Cesarianas: percepção de risco e sua indicação pelo obstetra em uma maternidade pública no Município do Rio de Janeiro}

\author{
Cesarean sections: risk perception and indication \\ by attending obstetricians in a public maternity \\ hospital in Rio de Janeiro
}

\author{
1 Secretaria Municipal \\ de Saúde do Rio de Janeiro, \\ Rio de Janeiro, Brasil. \\ 2 Pós-graduação em Saúde \\ da Criança e da Mulher, \\ Institu to Fernandes Figueira, \\ Fundação Oswaldo Cruz, \\ Rio de Janeiro, Brasil. \\ Correspondência \\ Marcos Augusto Bastos Dias \\ Rua Osório Duque Estrada 74 \\ Bl 2, 701, Rio de Janeiro, $R J$ \\ 22451-170, Brasil. \\ marcosad@centroin.com.br
}

\begin{abstract}
The cesarean section rate in municipal public maternity hospitals in the city of Rio de Janeiro in the year of 2000 was 30.1\%. With such a high rate, our hypothesis is that attending obstetricians in these units are indicating cesarean sections for reasons not restricted to clinical factors. The article aims to analyze physicians' representations of the inherent risks in cesarean sections that influence the medical decision for their indication. In this qualitative study, conducted through participant observation during ward duty and interviews with obstetricians, we observed that $C$-section indications by obstetricians are influenced by various non-obstetric factors, including insecurity about performing obstetric maneuvers, fragmented care, and fear of legal liability. The paper suggests that the widespread use of this intervention in private maternity services has fostered a practice in public obstetric services that compromises the quality of public health care and may jeopardize the health of women and infants.
\end{abstract}

Assistance; Parturition; Perception; Maternal and Child Health
Marcos Augusto Bastos Dias 1

Suely Ferreira Deslandes 2

\section{Introdução}

Ao longo do século XX, os inúmeros avanços da medicina transformaram a cesariana numa alternativa segura ao parto vaginal quando há algum risco para a mulher ou para o feto. Entretanto, desde a década de 70 , as indicações de cesariana vêm crescendo indiscriminadamente, e esta cirurgia sendo utilizada de forma abusiva em vários países 1,2 . Pelo seu uso indiscriminado, a cesariana tem se voltado contra os objetivos para os quais foi idealizada, ocasionando aumento nos riscos de morbimortalidade materna e perinatal 3 . No período de vinte anos compreendido entre 1970 e 1990, o grande aumento das taxas de cesariana em todo o mundo se transformou em foco de atenção não só pelo aumento da morbimortalidade materna e perinatal, mas também devido aos custos associados com o procedimento e à ausência de impacto na redução das taxas de perimortalidade 4 . No Brasil, este problema atingiu proporções ainda maiores e essas taxas que foram de 29,9\% em 1979 5, apesar das evidências científicas dos riscos atribuídos à esta cirurgia, continuaram a aumentar. Em uma recente avaliação das taxas de cesarianas de doze países latino-americanos, o Brasil apresentava a segunda maior taxa e os autores apontavam que outros fatores além das indicações clínicas exerciam um importante papel na distribuição desigual desta cirurgia nos diferentes 
extratos da população ${ }^{6}$. Um estudo ecológico sobre as taxas e as implicações das cesarianas na América Latina realizado por Belizan et al. 7 apontou uma correlação positiva e significativa entre o produto nacional bruto per capita e as taxas de cesariana, sendo que taxas maiores da cirurgia foram encontradas em hospitais privados do que em hospitais públicos. Os mesmos autores concluíram que além de representar um risco desnecessário para mulheres jovens e seus bebês, sob a perspectiva econômica esta situação comprometia ainda mais os sistemas de saúde com orçamentos limitados. Segundo o Sistema de Informação de Nascidos Vivos (SINASC), em 1999, as taxas de cesariana foram de $37,78 \%$ no Brasil; $42,25 \%$ na Região Sudeste e $47,93 \%$ no Estado do Rio de Janeiro. Cabe ressaltar que estes números ultrapassam, em muito, as recomendações da Organização Mundial da Saúde (OMS), que preconiza uma taxa máxima de $15 \%$ de cesarianas para qualquer país 8 .

As complicações maternas na cesariana podem variar de eventos menores como um episódio de febre ou a perda maior de volume de sangue, até eventos maiores como lacerações acidentais de vísceras, infecções puerperais e acidentes anestésicos 9 .

Em 1999, a taxa de cesariana no Município do Rio de Janeiro foi de 47\%, mas já em 1996, o Relatório de Trabalho no 2 da Gerência de Informação Epidemiológica da Secretaria Municipal de Saúde do Município do Rio de Janeiro, apontava que a prática abusiva desta cirurgia na proporção em que estava sendo utilizada, "aumentará a freqüência das complicações que poderão resultar desse tipo de procedimento" 10 (p. 2). Segundo este mesmo relatório, a freqüência de cesarianas foi maior nos estabelecimentos privados e para as mulheres com maior nível de instrução, reforçando a hipótese de Rattner 11 (p. 20), deste procedimento "ter se transformado em um bem de consumo, disponível para aqueles que detêm poder aquisitivo".

A Secretaria Municipal de Saúde do Rio de Janeiro (SMS-RJ) contava até 1999 com oito maternidades, sendo que em todas, mesmo naquelas que não são referência para gestantes de risco, nos últimos cinco anos, as taxas de cesariana ficaram acima dos níveis recomendados pela OMS 8. Tal fato, em tese, não deveria acontecer. Nestas maternidades o atendimento é gratuito e a remuneração dos profissionais não varia de acordo com o tipo de parto; os médicos trabalham em equipe o que teoricamente diminuiria os problemas relativos à deficiência na formação profissional de algum membro. Também o tempo de evolução do trabalho de parto não deveria pressionar os profissionais no sentido de acelerar o nascimento, já que os mesmos trabalham em regime de plantão, com horários determinados e os cuidados com a paciente serão continuados por uma nova equipe ao fim de sua jornada de trabalho.

As hipóteses que orientaram o estudo foram as de que as taxas de cesarianas das maternidades públicas são maiores do que as esperadas porque as indicações cirúrgicas são também regidas por questões ligadas à formação médica e às lógicas culturais da assistência.

Entender os mecanismos de decisão do médico obstetra ao indicar a interrupção da ges tação pela via abdominal e sua percepção dos riscos inerentes à esta cirurgia, poderá contribuir para que novas estratégias sejam desenhadas com o objetivo de reduzir as taxas de cesariana. Esta redução tornará possível não só oferecer uma assistência mais adequada e segura às mães e bebês, reorganizando os serviços para melhor atendê-los, como também racionalizar os gastos de recursos financeiros otimizando sua utilização.

Assim, este artigo tem como objetivo analisar as representações médicas sobre o risco inerente da cesariana, que além das indicações clínicas, influenciam as decisões médicas para a indicação das cesarianas.

\section{Metodologia}

Neste artigo, originário de uma pesquisa para Dissertação de Mestrado defendida junto ao Instituto Fernandes Figueira (IFF), Fundação Oswaldo Cruz (FIOCRUZ), optamos pela abordagem qualitativa para entender melhor se as representações do risco da cesariana e outros fatores não obstétricos influenciam os médicos no cotidiano da assistência numa maternidade pública e de que maneira as indicações cirúrgicas das cesarianas são construídas.

Nosso estudo toma de empréstimo a definição de representações sociais de Queiroz 12 (p. 27), entendendo-as como "um tipo de saber, socialmente negociado, contido no senso comum e na dimensão cotidiana, que permite ao indivíduo uma visão do mundo e o orienta nos projetos de ação e nas estratégias que desenvolve em seu meio social".

A concepção teórico-epistemológica adotada baseia-se no debate de Herzlich 13 com Moscovi sobre as representações sociais como um modo de pensamento ligado à ação, à conduta individual e coletiva. Concordamos com Herzlich sobre a crítica à idéia de uma causalidade mecânica entre representações sociais e uma 
conduta individual específica, sendo impossível e mesmo inadequado a estes estudos a previsão de quaisquer comportamentos individuais. Caberia, contudo, a busca de evidenciar os códigos culturais, situados num sistema de relações objetivas, contextualizados num dado momento da vida social, "a partir da qual se elaboram significações ligadas às condutas individuais e coletivas" 13 (p. 27).

As representações foram aqui delimitadas como opiniões e crenças dos médicos sobre os riscos e indicações do parto cesárea. Procuramos analisar de que maneira estão expressando o modo pelo qual os profissionais de saúde se organizam na assistência ao trabalho de parto e parto, se relacionam com as pacientes, entre si e com a instituição, e como todo este "complexo interativo" se reflete e interfere nas indicações da operação cesariana.

Seguindo a definição de Stake 14, consideramos este desenho como um estudo de caso instrumental, ou seja, quando o caso é analisado detalhadamente e em profundidade com vistas a estudo exploratório de uma determinada realidade ou situação, permitindo a elaboração de hipóteses. O caso pode ser visto como um "caso típico", representando um conjunto mais amplo, ou cujas particularidades permitem um debate sobre o objeto de estudo que se propõe. Embora tenha sido realizada uma etnografia da maternidade estudada, neste artigo discutiremos apenas as representações sociais dos médicos obstetras.

\section{O campo da pesquisa}

A maternidade escolhida para o trabalho de campo foi uma unidade da rede pública da SMS-RJ. O critério de escolha levou em conta o fato de a mesma não funcionar como referência para os atendimentos de situações de risco materno, nem estar localizada dentro de um hospital geral, características que poderiam estar influenciando as suas taxas de cesariana. Optamos, por cuidados éticos, em não identificar os profissionais e o nome da maternidade onde foi realizada a pesquisa. O estudo foi aprovado pelo Comitê de Ética em Pesquisa do IFF. Ao tratar desta maternidade, se por um lado traçamos um retrato que possui singularidades, por outro acreditamos que este cotidiano também espelha a realidade da assistência das maternidades públicas, bem como a lógica de ação de seus profissionais.

A população atendida nesta maternidade é em sua grande maioria de baixa renda e $80 \%$ das mulheres tinham baixa escolaridade. No ano de 1999 ocorreram nesta unidade cerca de
6.500 nascimentos vivos, dos quais $31,5 \%$ foram por cesariana.

Por serem responsáveis pela indicação e realização das cesarianas, os médicos obstetras (funcionários públicos concursados federais ou municipais) e os médicos residentes do programa de ginecologia e obstetrícia da maternidade foram os sujeitos pesquisados. Embora sejam todos médicos obstetras, pela diferença de vinte anos em média entre o tempo da graduação dos dois grupos (concursados e residentes), analisamos suas representações sociais em separado no sentido de avaliar se eram influenciadas pela diferença de gerações.

\section{Técnicas de pesquisa}

O trabalho de campo, na pesquisa qualitativa, é um "recorte espacial que corresponde à abrangência, em termos empíricos, do recorte teórico correspondente ao objeto da investigação" 15 (p. 109). Assim, em nosso trabalho de campo utilizamos as técnicas de observação participante e da realização de entrevistas. Entendemos que esses recursos seriam capazes de construir um acervo para a análise das representações dos profissionais médicos diante das indicações das cesarianas.

A definição de observação participante adotada enfatiza que a presença do observador numa situação social é mantida para fins de investigação científica. "O observador está em relação face a face com seus observados, e, em participando com eles em seu ambiente natural de vida, coleta dados. Logo, o observador é parte do contexto, sendo observado, no qual ele ao mesmo tempo modifica é modificado por este contexto" (Schwartz \& Schwartz, 1955, apud Haguette 16 p. 63).

A observação se deu com base em um roteiro. Acompanhamos o trabalho de uma mesma equipe durante os plantões, privilegiando as situações que faziam aflorar o encontro dos nossos pressupostos e marco teórico (o modelo de assistência e a banalização da indicação da cesariana) com os dados empíricos (indicações cirúrgicas por razões não relacionadas a patologias obstétricas). As observações, anotadas em diário de campo, aconteceram em 11 plantões nem sempre consecutivos. A decisão de encerrar o campo deu-se quando verificamos uma reincidência de situações que mostravam uma certa tendência. Neste trabalho, as observações de campo serviram para contextualizar e confrontar os relatos dos entrevistados.

A entrevista se mostra como um instrumento privilegiado para a análise qualitativa porque por meio da fala o entrevistado revela seus 
valores, sua percepção da realidade, explica e exemplifica a estrutura do seu sistema de referências e símbolos, transmitindo as representações de um grupo em determinado contexto cultural e sócio-econômico 15. Foram realizadas nove entrevistas semi-estruturadas com os médicos (efetivos e residentes) da equipe de plantão durante a pesquisa de campo. Os eixos temáticos foram: formação profissional; aprendizado de manobras obstétricas; diferenças na prática pública e privada; riscos relativos à cesárea. Optamos por fazer as entrevistas no final do período da observação participante, quando já tínhamos mais intimidade com os profissionais e observado suas práticas de assistência. Os médicos efetivos (referidos como médicos obstetras 1, 2, 3, 4 e 5), tinham idades entre 44 e 52 anos, sendo quatro homens e uma mulher. Os médicos residentes (identificados como residentes 1, 2, 3 e 4) tinham entre 25 e 27 anos, sendo três mulheres e um homem.

\section{A análise do material}

Como preocupação de toda interpretação qualitativa, buscamos avaliar se o que se percebeu estava verdadeiramente contido no material e se essa percepção não só era válida como também generalizável para o grupo estudado 17,18. Ao aprofundar a leitura do material, compreender seus significados e estruturas latentes foi possível juntar os dados desta leitura com o quadro de referência, contextualizando os aspectos envolvidos. Na prática, esta interpretação das falas dos obstetras e dos residentes levou à ordenação e classificação dos dados por unidade de registro, referenciadas por temas, agrupadas sob "núcleos de sentidos", cujas expressões de síntese, as categorias empíricas, foram trabalhadas. Por último, procedemos a análise final com o movimento de reflexão sobre os dados empíricos e o quadro teórico, quando procuramos buscar algumas hipóteses explicativas sobre os temas analisados 15 .

\section{Resultados e discussão}

Um questão inicial é a idéia de que as mulheres no Brasil preferem ter seus filhos por meio de uma cesariana pelo medo da dor no parto vaginal, para preservar a função sexual e para a realização da laqueadura tubária, entre outros 1. Embora as taxas de cesariana no Brasil sejam extremamente elevadas, elas nem sempre refletem o desejo das mulheres quanto ao tipo de parto que preferem. Vários autores têm des- mistificado esta noção, apontando a preferência das mulheres pelo parto vaginal, tanto aquelas assistidas nos serviços públicos quanto nos privados 19,20.

A percepção reinante sobre cesarianas entre os profissionais estudados, técnicos treinados que as executam dezenas de vezes, é de que são cirurgias seguras, rápidas e sem complicações, melhores para as mulheres, para os bebês e também para os médicos. Não haveria problemas em se indicar cesarianas de forma liberal sem seguir estritamente apenas os critérios obstétricos. Quem estaria sendo prejudicado? Ao realizar uma cesariana, o médico estaria não apenas salvando a vida da mãe e do feto, mas também abreviando o sofrimento da mulher ocasionado pelas contrações do trabalho de parto. A liberalidade nas indicações de cesariana também é favorecida porque apesar de saber que, do ponto de vista teórico, as cesarianas têm mais complicações tanto maternas quanto fetais, eles não identificam esse risco na sua prática diária.

"Eu acho que pode ser uma coisa contraditória, é real, o risco da cesárea é maior do que a morbidade de um parto normal, isso todo mundo sabe, do que um parto literalmente normal, não é?" (Médico 2).

Os médicos, principalmente os obstetras do quadro, minimizam os riscos das cesarianas, uma vez que os mesmos, na maior parte, não acompanham o pós-operatório das pacientes que operaram durante o plantão ou para as quais indicaram a cirurgia. Quando retornam à maternidade no plantão seguinte não têm mais nenhuma notícia do que aconteceu à paciente, nem tem o hábito de informar-se. Essa percepção da ausência de risco, principalmente em relação à infecção no pós-operatório, colide com os depoimentos dos médicos residentes que como são responsáveis pelo acompanhamento das pacientes, apontam uma incidência grande de infecções cirúrgicas.

“...Risco de infecção está bastante controlado, você vê, pelo menos aqui na instituição; na clínica privada, eu nunca tive uma infecção, graças a Deus,espero não ter, é nunca tive, aqui... quer dizer, nossos casos atualmente, não tem tido, praticamente acho que não tem, não está tendo risco. E o pessoal, quer dizer: sabe operar. Sabe operar. O risco maior seria de quê? De uma infecção, basicamente, e não tem tido." (Médico 1).

"A existência do risco de infecção, que é o que eu mais vejo, não posso falar da parte fetal, neonatal, não tenho muita experiência nisso, mas parte materna, infecção, aqui a gente vê bastante..." (Residente 1). 
Fica evidente a correlação entre a prática obstétrica realizada na clínica privada e no serviço público, embora a população em termos sócio-econômicos seja bastante distinta e as cesarianas na clínica privada sejam realizadas em sua grande maioria de forma eletiva, fator importante na ocorrência de complicações operatórias da cesariana 9,21 .

A existência do risco "real" da cesariana também é relativizada pelos profissionais que o contrapõe aos riscos do parto vaginal, diminuindo desta forma uma possível “culpa” por estar submetendo a mulher a um procedimento desnecessário.

"Não, eu não verifico este risco, eu acho inclusive que o parto transvaginal, ele também tem os seus riscos, tem suas complicações hemorrágicas, suas complicações infecciosas, as complicações de puerpério imediato, é maior quando ele é mal conduzido, você tem todo risco fetal em que... na cesárea se você tiver uma indicação precisa, se você tiver todo um cuidado, diminui. Eu acho que aumenta, eu acho que o risco existe na... das duas maneiras, tanto transvaginal como via alta" (Médico 3).

A própria deficiência na formação obstétrica serve como justificativa para ampliar as indicações de cesariana. Como são muitas as cesarianas indicadas, não existe a possibilidade de que um residente não tenha a oportunidade de realizar várias destas cirurgias durante o seu primeiro ano do programa de residência. Em nenhuma oportunidade durante o trabalho de campo qualquer médico residente do segundo ano disse ter qualquer dificuldade em realizar uma cesariana. Por outro lado, as manobras obstétricas de assistência ao parto pélvico, ao parto gemelar ou mesmo a aplicação de fórceps são realizadas raramente, fazendo com que os residentes e os próprios médicos obstetras tenham cada vez menos a oportunidade de praticá-las, ao contrário da cesariana, para a qual existem várias oportunidades de treinamento. Embora todos os profissionais considerem importante o aprendizado das manobras obstétricas, nem todos se sentem seguros em realizá-las.

"...Você tem que saber, você pode até não usar com muita freqüência, mas tem que saber fazer, para se você precisar você poder botar a mão nisso..." (Residente 3).

“...Eu acho que o risco maior da cesárea é o risco anestésico, que está igual ao do parto normal, se você for considerar o parto com analgesia, praticamente o mesmo, porque habilidade cirúrgica, quer dizer: todo mundo agora... só faz basicamente cirurgia, então está todo mundo sabendo operar, fazer uma cesárea direitinho. Eu acho que risco...não..." (Médico 1).
A oportunidade do aprendizado, pelos médicos residentes, destas manobras também é influenciada pela capacidade dos médicos obstetras de realizá-las e pela maneira como estes julgam este tipo de assistência. Se o médico é habilitado na operatória obstétrica e considera sua prática efetiva, ele vai transmitir esses conhecimentos ao residente. Se não tiveram a formação ou não têm a segurança para a sua prática, não utilizarão tais manobras no seu trabalho diário e, portanto, não terão como ensinar estas manobras aos residentes.

“...Numa época em que o parto vaginal era a coisa mais importante. A cesárea era...não que não fosse feita, era feita, mas quando realmente existia uma indicação, ou materna ou fetal real, que aprendi a passar o fórceps... o parto pélvico, nós fazíamos partos pélvicos...." (Médico 1).

Como os casos em que é necessária a realização de manobras obstétricas estão associados a um maior risco fetal e materno, muitas vezes opta-se pela realização da cesariana com a intenção de não correr este risco. Com esta conduta e com o aumento da segurança na realização da cesariana, cada vez menos os profissionais sentem-se capacitados e confiantes para realizar estas manobras alternativas, optando pela cirurgia ao menor sinal de que o trabalho de parto não está absolutamente sob controle e alguma complicação que eles não saibam resolver possa acontecer. O mesmo profissional que afirmava como a sua formação havia lhe capacitado para a operatória obstétrica justifica a sua pouca utilização pelo medo de complicações e da possibilidade de processos éticos ou judiciais.

“...Eu acho que a obstetrícia está mudando, eu não sei se é medo das complicações, ou falta de formação mesmo, entendeu?... Você é pressionado porque vai ter um processo contra você, você tem que acertar sempre, você...sabe...não pode haver nenhuma complicação, em decorrência disso você modifica até suas condutas, como eu também modifico, com medo de uma pressão externa, então é medo, é pressão, por isso, porque eu vejo, que o índice de cesáreas aumentou no Brasil... você não vai se expor, eu não tenho necessidade agora de me expor, para quê??" (Médico 1).

Este círculo vicioso faz com que as manobras de operatória obstétrica e o parto a fórceps sejam utilizados com menor freqüência. Com o passar do tempo, um número menor de profissionais estará habilitado para realizá-las e conseqüentemente ensiná-las aos novos obstetras, tendendo estas condutas a desaparecer da prática obstétrica, diferentemente do que ocorre em centros obstétricos de excelência do 
primeiro mundo, onde estas manobras são praticadas rotineiramente. Para Gei \& Belfort 22, embora o uso do parto vaginal esteja em declínio, a habilidade de aplicar o fórceps obstétrico de modo seguro permanece como um importante predicado que diferencia os obstetras dos cirurgiões, e continua a ter um importante lugar na obstetrícia moderna.

"Eu acho que vai acabar...acho...infelizmente. Porque está faltando formação, aqueles que fazem estão acabando, estão se aposentando, estão morrendo, e não estão passando, em decorrência das condições institucionais, vamos dizer, desse medo, dessa coisa, dessa pressão, não estão passando para os outros, para os que estão começando, e os que estão começando...não conhecem, entendeu? Então eu acho que futuramente não vai existir" (Médico 1).

Hotimsky et al. 20 apontam que a formação dos obstetras vem ocorrendo em meio a uma cultura médica que tem privilegiado o uso de equipamentos sofisticados em detrimento da adoção das tecnologias apropriadas à assistência perinatal, prevalecendo uma visão de risco em relação aos eventos fisiológicos da gestação e do parto.

A visão dos médicos obstetras e dos residentes é de que a cesariana seria uma forma de "profilaxia das complicações" que não saberiam manejar, de um acompanhamento clínico que não poderiam oferecer e de evitar processos por conta de mal resultados. Aparentemente sem riscos e com poucas chances de complicar, seria mais "seguro" fazer uma cesariana.

"Aqui acho que é mais social, quer dizer, eu acho que a maioria das indicações aqui, são indicações sociais ou indicações profiláticas...indicação social é...vamos dizer, é a paciente pobre, paciente multípara, entendeu? Então muitas vezes faz-se a cesárea para ligadura,entendeu? É aquilo que eu digo assim: indicação profilática, é profilaxia da complicação que o obstetra não sabe solucionar, que é a operatória obstétrica, entendeu? Então existem muitas indicações assim" (Médico 1).

As diferentes taxas de cesarianas nos vários países mostram que a indicação desta cirurgia maior, pelos obstetras, nos diferentes hospitais e nas sociedades em que eles exercem sua prática está sujeita a diferentes protocolos. Para Enkin et al. 23 essas indicações estão sujeitas ao status sócio-econômico da mulher, a questões financeiras, de processos judiciais, de conveniência da mulher e do profissional e que podem ser algumas vezes mais importantes que os fatores obstétricos.

\section{Conclusões}

Ao estudar as representações sociais destes profissionais, não há como deixar de considerar todo o contexto sociocultural que os envolve, e que é por eles invocado para justificar sua prática. Em que pese os questionamentos apresentados sobre a construção das indicações da operação cesariana, muito provavelmente a atuação destes profissionais tem como objetivo o que pelo senso comum seria o melhor para as mulheres e seus bebês.

As indicações das cesarianas vão sendo banalizadas na medida em que as representações de risco dos médicos obstetras vão se consolidando em rotinas ao longo da sua formação e experiência profissional no que tange à assistência ao parto. Na diferença de cerca de 20 anos entre estas duas gerações (médicos do staff e médicos residentes), deu-se o abandono das técnicas de operatória obstétrica transpélvica e sua substituição, na resolução das complicações do trabalho de parto, pela cesariana. $\mathrm{O}$ ensino e a prática destas técnicas foram praticamente extintos, deixando os novos profissionais desarmados para enfrentar situações em que sua utilização evitaria a cesariana. A insegurança na realização destas manobras associada ao receio de um processo judicial, faz com que sua utilização esteja reservada às situações desesperadoras, quando não há mais a possibilidade de realizar uma cesariana e, portanto com grandes chances de um mal resultado neonatal. Como na análise dos dados neonatais desfavoráveis, resultantes dos partos vaginais, levanta-se quase sempre a possibilidade de que uma cesariana poderia ter evitado o desfecho negativo e em geral não se avaliam rotineiramente as complicações maternas ou fetais decorrentes de uma cesariana realizada desnecessariamente, reitera-se a idéia de que é mais seguro realizar a cirurgia.

A fragmentação da assistência obstétrica faz com que o médico plantonista não acompanhe o pós-operatório das pacientes por ele operadas. Assim, o mesmo não tem uma idéia das complicações que acontecem no período pós-operatório, reforçando a falsa idéia de segurança da cesariana. Da mesma forma parecem desconhecer que as cesarianas realizadas durante o trabalho de parto (maior parte dos casos no serviço público) apresentam efetivamente um risco maior do que aquelas realizadas de forma eletiva e que são a experiência predominante na clínica privada da maior parte dos profissionais. A cesariana é vista não apenas como uma ferramenta poderosa, que poderia resolver todas as situações passíveis de 
complicação e que os profissionais se sentem mais do que capacitados para realizá-la, como também funciona como a profilaxia de possíveis complicações éticas ou judiciais. A cesariana é a ação médica que consegue dar um desfecho previsível ao trabalho de parto, uma situação que pelas suas características originais, não se definiria até o último instante com o nascimento do bebê.

Consideramos que é preciso mudar o paradigma do modelo médico de nascimento exclusivamente técnico, que deixa de valorizar os aspectos sociais e culturais da gestação e do parto, por um modelo que sem abrir mão das conquistas da medicina moderna permita um resgate destes aspectos tão importantes. Acreditamos que a formação médica tem papel fundamental nesta mudança, e que deva incorporar, entre outros, conceitos da bioética, das ciências sociais e seus conteúdos humanísticos, permitindo ao profissional um novo olhar para estes eventos. Na busca de soluções para esta mudança devemos considerar as recomendações da OMS 8 e principalmente as evidências científicas que devem embasar nossas decisões. Devolver à mulher o papel de sujeito do parto, respeitar a fisiologia do trabalho de parto, oferecer o devido suporte à mulher e à sua família. Este parece ser o caminho para uma assistência que terá melhores resultados expressos não apenas nas taxas de mortalidade materna e perinatal, como também na satisfação das mulheres e dos profissionais de saúde. Esta difícil tarefa, que resultará certamente na redução das taxas de cesariana, deve estar a cargo de toda a sociedade e não apenas dos profissionais de saúde. É preciso que, juntos, reconstruamos o senso comum que hoje transformou o parto normal em sinônimo de sofrimento e a cesárea em uma mercadoria milagrosa e sofisticada, que pode ser vendida e comprada sem maiores conseqüências.
Resumo

A taxa de cesariana entre as maternidades públicas municipais da cidade do Rio de Janeiro no ano de 2000 foi de 30,1\%. Com taxas tão elevadas, nossas hipóteses são que as indicações de cesarianas nestas unidades não estão restritas apenas às indicações clínicas. Este artigo tem como objetivo analisar as representações de médicos sobre os riscos inerentes da cesariana que influenciam a sua indicação. Neste estudo qualitativo, realizado com observação participante de plantões e entrevistas com obstetras, pudemos identificar que as indicações feitas pelos obstetras da unidade pública estudada sofrem influência de diversos fatores não obstétricos: a insegurança quanto às manobras obstétricas, a fragmentação do atendimento e o medo da responsabilização jurídica, entre outros. $O$ artigo sugere que a banalização desta intervenção no serviço privado traz para o serviço público um desvio da prática obstétrica que compromete não apenas a qualidade da assistência pública, como pode colocar em risco a vida de mulheres e bebês.

Assistência; Parto; Percepção; Saúde Materno-Infantil

\section{Colaboradores}

M. A. B. Dias contribuiu com a realização da pesquisa e redação do artigo. S. F. Deslandes colaborou na redação do artigo. 


\section{Referências}

1. Faundes A, Ceccatti JG. A operação cesárea no Brasil. Incidência, tendências, causas, conseqüências e propostas de ação. Cad Saúde Pública 1991; 7:150-73.

2. Bottoms SF, Rosen MG, Sokol RJ. Increase in the cesarean birth rate and its implications for the practice of obstetrics. In: Osofsky HJ, editor. Advances in clinical obstetrics and gynecology. v.1. Baltimore: Williams \& Wilkins; 1981. p. 115-32.

3. O'Driscoll K, Foley M. Correlation of decrease in perinatal mortality and increase in cesarean sectionrates. Obstet Gynecol 1983; 61:1-5.

4. Shearer EL. Cesarean section: medical benefits and costs. Soc Sci Med 1983; 37:1223-31.

5. Saas N, Souza E, Camano L. Operação cesariana. Análise crítica dos índices. Gynaecia 1997; 3:27-34.

6. Béhague DP, Victora CG, Barros FC. Consumer demand for caesarean sections in Brazil: informed decision making, patient choice, or social inequality? A population based birth cohort study linking ethnographic and epidemiological methods. Br Med J 2002; 324:942-5.

7. Belizán JM, Althabe F, Barros FC, Alexander S, Showalter E, Griffin A, et al. Rates and implications of cesarean sections in Latin America: ecological study. Br Med J 1999; 319:1397-402.

8. Organização Mundial da Saúde. Care in normal birth; a pratical guide, maternal and newborn health/safe motherhood unit. Family and reproductive health. Geneva: World Health Organization; 1996.

9. Van Ham MA, Van Dongen PW, Mulder J. Maternal consequences of cesarean section. A retrospective study of intra-operative and postoperative maternal complications of cesarean section during a 10-year period. Eur J Gynecol Reprod Biol 1997; 74:1-6.

10. Secretaria Municipal de Saúde do Rio de Janeiro. Relatório de trabalho no 2. Sistema de informação sobre nascidos vivos - SINASC 1993/1996. Rio de Janeiro: Secretaria Municipal de Saúde do Rio de Janeiro; 1997

11. Rattner D. Sobre a hipótese de estabilização das taxas de cesáreas do Estado de São Paulo, Brasil. Rev Saúde Pública 1996; 30:19-33.
12. Queiroz MS. Representações sociais: uma perspectiva multidisciplinar em pesquisa qualitativa. In: Barata RB, Briceño-León R, organizadores. Doenças endêmicas. Abordagens sociais, culturais e comportamentais. Rio de Janeiro: Editora Fiocruz; 2000. p. 27-46.

13. Herzlich C. A problemática da representação social e sua utilidade no campo da doença. Physis (Rio J) 1991; 1:23-36.

14. Stake RE. Case Studies. In: Denzin NK, Lincoln YS, editors. Handbook of qualitative research. London: Sage Publications; 2000. p. 435-54.

15. Minayo MCS. O desafio do conhecimento. Pesquisa qualitativa em saúde. São Paulo: Editora Hucitec/Rio de Janeiro: ABRASCO; 1992.

16. Haguette TMF. Metodologias qualitativas na sociologia. Petrópolis: Editora Vozes; 1987.

17. Deslandes SF, Assis SC. Abordagens quantitativa e qualitativa em saúde: o diálogo das diferenças. In: Minayo MCS, Deslandes SF, organizadoras. Caminhos do pensamento. Epistemologia e método. Rio de Janeiro: Editora Fiocruz; 2002. p. 195-223.

18. Le Compte MD. Problems of reliability and validity in ethnographic research. Review of educational research 1982; 52:31-60.

19. Potter JE, Berquó E, Perpetuo IHO, Leal OF, Hopkins K, Souza MR, et al. Unwanted cesarean sections among public and private patients in Brazil: prospective study. Br Med J 2001; 323:1155-8.

20. Hotimsky SN, Ratnner D, Venancio SI, Bógus CM, Miranda MM. O parto como eu vejo... ou como eu desejo? Expectativas de gestantes, usuárias do SUS, acerca do parto e da assistência obstétrica. Cad Saúde Pública 2002; 18:1303-11.

21. Lilford RJ, Van Coeverden-de Groot HA, Moore PJ, Bingham $P$. The relative risks of cesarean section (intrapartum and elective) and vaginal delivery: a detailed analisys to exclude the effects of medical and other acute pre-existing physiological disturbances. Br J Obstet Gynaecol 1990; 97:883-92.

22. Gei AF, Belfort MA. Forceps-assisted vaginal delivery. Obstet Gynecol North Am 1999; 26:345-70.

23. Enkin M, Keirse MJNC, Neilson J, Crowther C, Duley L, Hodnett E, et al. A guide to effective care in pregnancy and childbirth. New York: Oxford University Press; 2000.

Recebido em 09/Mai/2003

Versão final reapresentada em 20/Ago/2003 Aprovado em 04/Set/2003 